\title{
OBSERVATIONS OF SUPERNOVA REMNANTS WITH ISO
}

\author{
R.J. TUFFS \\ Max-Planck-Institut für Kernphysik \\ Postfach 103980 D-69117 Heidelberg, Germany
}

\begin{abstract}
Pointed observations with ISO have been made, or are planned, towards 58 supernova remnants (SNRs) in the galaxy and Magellanic clouds. The main emphasis is on detailed studies of gas, dust and synchrotron emission from selected regions within 24 well studied galactic remants. First results are available for Cas A (Lagage et al. 1996, 1997; Tuffs et al. 1997; Unger et al. 1997), the Cygnus Loop (deCourchelle et al 1997; Fischera et al. 1997), RCW103 (Oliva et al. 1997; Wright et al. 1997), and W44 and 3C391 (Reach and Rho 1996). These are providing insights into issues such as the yield and composition of condensates in supernova ejecta, the processing of grains by adiabatic and radiative shocks, and the measurement of cold gas, inaccessible to optical observations, both from cool regions in radiative shocks and from unshocked ejecta. In this progress report these points are illustrated with reference to Cas A, the prototype of an ejecta-dominated remnant of a massive core-collapse supernova, and the Cygnus Loop and RCW103 as prime examples of radiative shocks delineating the interaction of fast blast waves with diffuse atomic and dense molecular clouds, respectively.
\end{abstract}

\section{Cassiopeia A}

The ISOCAM 10.7-12 $\mu \mathrm{m}$ image of Cas A from Lagage et al. (1996), reproduced Fig. 1, shows a clumpy shell, broadly coincident with the radio and X-ray shell, superimposed on which are compact structures similar to the pattern of optical emission lines from ballistically moving supernova ejecta (the fast optical filaments). Spectrophotometric mapping of the northern shell by Lagage et al. (1997) with a CVF scan between 5 and $15 \mu m$ at $\Delta \lambda / \lambda \sim 40$, and by Tuffs et al. (1997) at $\Delta \lambda / \lambda \sim$ 100 in the 2.5-11.6 $\mu \mathrm{m}$ range using ISOPHOT-S reveal strong doppler broadened emission near [ArII] $6.99 \mu \mathrm{m}$, [Ar III] $8.99 \mu \mathrm{m}$, [S IV] $10.5 \mu \mathrm{m}$ and [Ne II] $12.8 \mu \mathrm{m}$, accounting for about half the MIR luminosity of the northern shell shortwards of $13 \mu \mathrm{m}$. The space-velocity distribution of these dominant MIR lines is very similar to the pattern seen in [S II] $\lambda \lambda 6716,6731$ and [Ar III] $\lambda \lambda 7136$, implying rather uniform excitation and relative abundances for the ejected Ar, S and Ne. However, ISOPHOT-S also detected faint emission from [Co II] $(2.99$ and $10.52 \mu \mathrm{m})$, [Ni I] $(3.12$ and $3.95 \mu \mathrm{m})$ and [Fe II] 4.08, 4.11, 4.43, 4.61 and $4.67 \mu \mathrm{m}$, indicating that the space-velocity pattern of iron group elements differs from that of $\mathrm{Ar}, \mathrm{S}$ and $\mathrm{Ne}$.

The MIR spectrum of Cas A is very rich and continuum emission is spectrally confused with fine structure, molecular and solid state emission features over much of the wavelength range of ISOPHOT-S. A faint underlying continuum can nevertheless be discerned longwards of $\lambda \sim 7 \mu \mathrm{m}$, with a broad bump from $8-11 \mu \mathrm{m}$ which may plausibly be identified with silicate. The morphological similarity between ISOCAM CVF images at $\lambda 9.8 \mu \mathrm{m}$ and in the [S IV] and [Ne II] lines delineating ejecta thus provides convincing evidence for the presence of condensates in the supernova ejecta (Lagage et al. 1996), the first such detection in a galactic SNR. Also seen in the ISOPHOT$\mathrm{S}$ spectra are precursor molecules of the condensates, notably $\mathrm{SiO}$ around $8.05 \mu \mathrm{m}$, raising the prospect that deeper observations could measure isotope ratios in the ejecta. In common with the other SNRs observed so far, none of the unidentified emission features commonly attributed to PAHs are apparent in the spectra towards Cas A.

The brightness of the diffuse MIR counterpart to the radio and X-ray shell declines dramatically with decreasing wavelength, consistent with a paucity of emission from grains with temperatures $>200 \mathrm{~K}$ (with sizes $<50 \mathrm{~nm}$ ). The brightness of the shell measured by ISOPHOT in a spectral region apparently free of spectral features around $6 \mu \mathrm{m}$ is comparable with the extrapolation of 


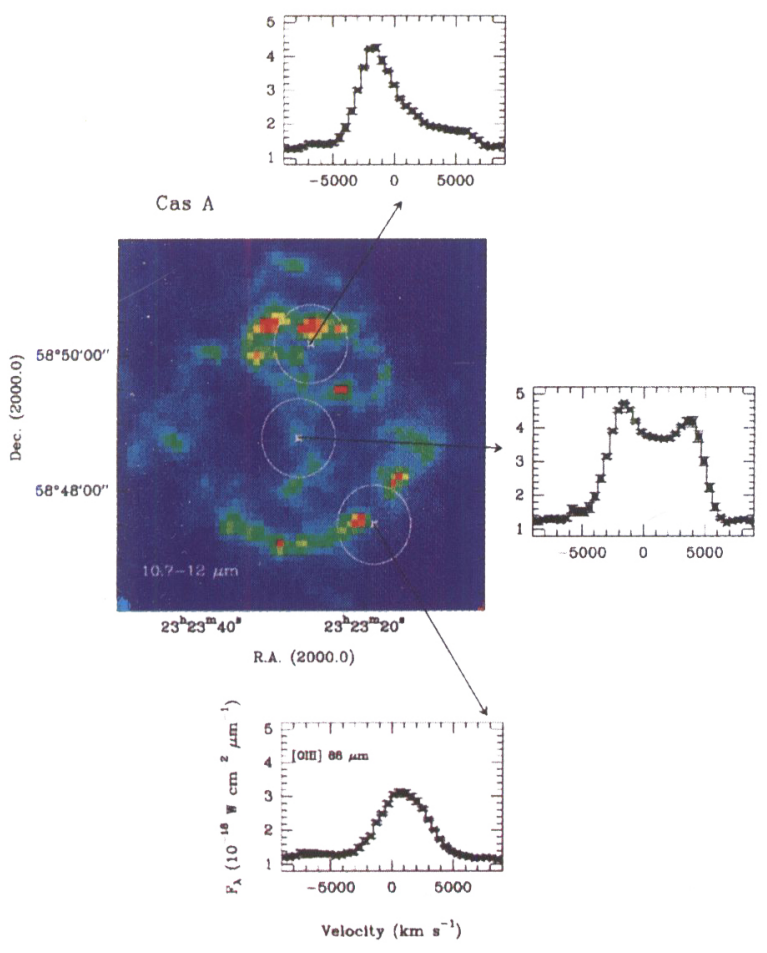

Figure 1. The ISOCAM 10.7-12 $\mu \mathrm{m}$ image of Cas A, observed with $6^{\prime \prime}$ pixels, reproduced from Lagage et al. (1996). The circles denote the LWS aperture at the positions observed with LWS by Unger et al. (1997). The three spectra shown are of the background subtracted [O III] $88 \mu \mathrm{m}$ ]ine

the radio synchrotron spectrum, consistent with the identification of the $10-100 \mathrm{keV}$ spectrum as synchrotron emission by Allen et al. (1997).

FIR mapping of Cas A in the 60-200 $\mu m$ range (Tuffs et al. 1997) places an upper limit of $\sim 0.05 \mathrm{M}_{\odot}$ on the mass of cold grains in the metal-rich unshocked ejecta interior to the shell. This is much less than the theoretical maximum yield of several solar masses (perhaps implying the ejecta were chemically unmixed), suggesting that very massive core-collapse supernovae are unimportant as sources of interstellar grains. By contrast, the unshocked ejected gas may have been detected by LWS (Unger et al. 1997). The LWS spectra reproduced in Fig. 1 shows velocity broadened profiles in [O III] $\lambda 88 \mu \mathrm{m}$ in the northern (blueshifted) and south-eastern (redshifted) parts of the shell, similar to the velocity profiles associated with the shocked fast optical filaments measured by Lawrence et al. (1995). Towards the centre of the remnant, however, where there is only faint optical emission, the [O III] $\lambda 88 \mu m$ emission is comparably bright to that seen in the shell, with radial velocities within the $\pm 5000 \mathrm{~km} \mathrm{~s}^{-1}$ range expected for freely expanding cold ejecta interior to the reverse shock. This may be the emission counterpart to the cold ionized gas responsible for the low frequency radio free-free absorption within the central 1' (Kassim et al. 1995), though the heating source for the central [O III] $\lambda 88 \mu m$ emission is not obviously apparent. 


\section{The Cygnus Loop and RCW103}

The Cygnus Loop is rather faint in the MIR and it is consequently even more of a challenge to separate the gaseous line emission from the dust continuum than for Cas A. ISOCAM mapping has been made of a field centred on the brightest X-ray emission (Eastern Knot) in the Loop by deCourchelle et al (1997) using the $\lambda 8.5-10.710 .7-12$ and 12-18 $\mu \mathrm{m}$ filters. Clear emission was detected only in the $12-18 \mu \mathrm{m}$ band, morphologically uncorrelated with the X-ray emission but becoming increasingly well correlated with optical line emission tracing progressivly colder gas. The most straightforward explanation is that the MIR emission in this region is dominated by line emission from [NeII] $12.8 \mu \mathrm{m}$ and [Ne III] $15.6 \mu \mathrm{m}$ from the radiative shocks.

In a complementary study of a field centred on the brightest IRAS peak in the North East of the Loop, Fischera et al. (1997) mapped emission in the $\lambda 5-8.5,8-15$ and $12-18 \mu \mathrm{m}$ filters with ISOCAM and in the $\lambda 40-80$ and $80-120 \mu \mathrm{m}$ filters using ISOPHOT-C, finding a very similar morphology to the optical [O III], $\left[\mathrm{SII}_{j}\right.$ and $\mathrm{H}_{\alpha}$ emission from the filaments in all three bands. No MIR emission was detected from the diffuse X-ray component associated with the blast wave in the intercloud medium. However, preliminary results from ISOPHOT-P photometry in a selection of narrow and broad-band filters tentatively suggest that the bulk of the emission associated with the filaments in this field is actually from a dust continuum, even at the shortest wavelengths around $\lambda 7 \mu \mathrm{m}$. If so, the MIR colours imply a surfeit of small grains compared with DIRBE measurements of the cold diffuse interstellar medium by Bernard et al. (1994). The source of this population of small grains might plausibly be grain-grain collisions in the cool and dense regions of the shocks.

In RCW103 the interaction of the blast wave with the dense molecular material is stronger, providing enough IR brightness for grating spectroscopy. Full SWS and LWS spectral scans taken towards the peak of the [Fe II] $1.644 \mu \mathrm{m}$ line emission interior to the shell of strong $\mathrm{H}_{2} \mathrm{NIR}$ emission by Oliva et al. (1997) are line dominated, showing very little continuum at $\lambda<40 \mu \mathrm{m}$. Gas phase $\mathrm{Fo}$ and $\mathrm{Si}$ abundances derived from the FIR spectra are close to solar relative to non-refractory species such as $\mathrm{Ne} \mathrm{S}$ and Ar, suggesting that the shock has been effective in destroying the interstellar dust. This is qualitatively consistent with the absence of a MIR dust continuum.

One question addressed by ISO concerns the excitation mechanism for the $\mathrm{H}_{2}$ line emission from RCW103. Wright et al. (1997) observed the molecular hydrogen peak with SWS in a series of rotational and rotational-vibrational $\mathrm{H}_{2}$ lines and derived excitation diagrams ( $\log$ (column density/statistical weights) versus upper level energy) from extinction corrected line fluxes. The preliminary indication is that the RCW103 excitation diagrams are more similar to those seen in sources known to be shock excited, such as the protostellar outflow source Cep A (Wright et al. 1996), rather than sources excited by UV fluorescence. There is also an indication of brighter emission from the lowest pure rotational transition at $28.2 \mu \mathrm{m}$ than predicted from the extrapolation of the higher rotation transitions. This hints that there may be a significant amount of cool $(<200 \mathrm{~K})$ gas upstream of the putative shock.

\section{Acknowledgements}

I thank Anne deCourchelle, Pierre-Oliver Lagage, Tino Oliva, Sarah Unger and Chris Wright for communicating results prior to publication.

\section{References}

1. Allen, G.E. et al. (1997). Astrophys. J. Lett., Vol. no. 487, p. L97

2. Bernard, J.P. et al. (1994). Astron. Astrophys, Vol. no. 291, pp. L5-L8.

3. deCourchelle, A., J-L. Sauvageot and W. Reach (1997) in preparation.

4. Fischera, J. et al. (1997) in preparation.

5. Kassim et al. (1995). Astrophys. J. Lett., Vol. no. 455, pp. L59-62.

6. Lagage, P.O et al. (1996). Astron. Astrophys, Vol. no. 315, pp. L273-276

7. Lagage, P.O. et al. (1997) in proc. "ISO's View on Stellar Evolution".

8. Lawrence, S.S et al. (1995). Astron. J., Vol. no. 109, p. 2635

9. Oliva, E. et al. (1997). in proc. "ISO's View on Stellar Evolution"

10. Reach, W.T. and Rho, J. (1996). Astron. Astrophys, Vol. no. 315, pp. L277-280

11. Tuffs, R.J. et al. (1997) in preparation.

12. Unger, S. et al. (1997) in preparation.

13. Wright, C., Drapatz, S. and Timmermann, R. (1996). Astron. Astrophys, Vol. no. 315, pp. L301.

14. Wright, C. et al. (1997) in preparation. 